\title{
Solution of Maxwell's equations
}

\author{
Michael Bartsch a , Micha Dehler a, Martin Dohlus a , Frank Ebeling a , Peter \\ Hahne $^{\text {a }}$, Reinhard Klatt ${ }^{a}$, Frank Krawczyk ${ }^{a}$, Michaela Marx ${ }^{b}$, Zhang Min ${ }^{a}$, \\ Thomas Pröpper ${ }^{\mathrm{a}}$, Dietmar Schmitt ${ }^{\mathrm{a}}$, Petra Schütt ${ }^{\mathrm{a}}$, Bernhard Steffen ${ }^{\mathrm{c}}$, \\ Bernhard Wagner $^{\text {a }}$, Thomas Weiland ${ }^{\text {a }}$, Susan G. Wipf ${ }^{\mathrm{b}}$ and Heike Wolter ${ }^{\mathrm{a}}$ \\ a Technische Hochschule Darmstadt, Fachbereich 18, Fachgebiet Theorie Elektromagnetischer Felder, \\ Schloßgartenstraße 8, W-6100 Darmstadt, Germany \\ b Deutsches Elektronen-Synchrotron DESY, Notkestraße 85, W-2000 Hamburg 52, Germany \\ ' Kernforschungsanlage Jülich KFA, W-5170 Jülich, Germany
}

\begin{abstract}
A numerical approach for the solution of Maxwell's equations is presented. Based on a finite difference Yee lattice the method transforms each of the four Maxwell equations into an equivalent matrix expression that can be subsequently treated by matrix mathematics and suitable numerical methods for solving matrix problems. The algorithm, although derived from integral equations, can be considered to be a special case of finite difference formalisms. A large variety of two- and three-dimensional field problems can be solved by computer programs based on this approach: electrostatics and magnetostatics, low-frequency eddy currents in solid and laminated iron cores, high-frequency modes in resonators, waves on dielectric or metallic waveguides, transient fields of antennas and waveguide transitions, transient fields of free-moving bunches of charged particles etc.
\end{abstract}

\section{Introduction}

The field of accelerator physics largely deals with controlled application of electromagnetic forces to charged particles. These forces occur in various parts of an accelerator at different levels of complexity: Magnetostatic and electrostatic fields are used to steer, focus and accelerate particle beams. RF-fields in metallic resonators are the most common type of accelerating devices. CW-transmitters in the UHF range of more than $1 \mathrm{MW}$ output power are used in large numbers, pulsed tubes are being built up to $100 \mathrm{MW}$ in the S-band. Transient fields are excited by charged particles when passing accelerator structures and cause difficult nonlinear problems (collective effects).

In the field of elementary particle physics accelerators as large as 27 kilometers in circumference are commissioned and plans for machines beyond 180 kilometers have been presented. The cost of these front-line accelerators is enormous. Thus it is extremely important to ensure, prior to construction, that such a device will work as planned. In order to predict the behaviour of charged-particle beams in accelerators, one has to solve a variety of field problems for realistic structures such as those mentioned above. This has occasioned the developement of algorithms and program systems, which have already proved dependable and useful in many areas of physics and engineering.

Correspondence to: T. Weiland, Technische Hochschule Darmstadt, Fachbereich 18, Fachgebiet Theorie Elektromagnetischer Felder, Schloßgartenstraße 8, W-6100 Darmstadt, Germany 


\section{The method}

In order to avoid specializations of Maxwell's equations prior to numerical solution it is advantageous to solve Maxwell's equations directly, rather than solving a partial differential equation derived therefrom. Using SI units, $\boldsymbol{E}$ and $\boldsymbol{H}$ for the electric and magnetic field strength, $\boldsymbol{D}$ and $\boldsymbol{B}$ for the flux densities and $J$ for the current density the equations to solve read

$$
\begin{aligned}
& \oint_{\partial \boldsymbol{A}} \boldsymbol{E} \cdot \mathrm{d} \boldsymbol{s}=-\iint_{\boldsymbol{A}} \frac{\partial \boldsymbol{B}}{\partial t} \cdot \mathrm{d} \boldsymbol{A}, \\
& \oint_{\partial A} \boldsymbol{H} \cdot \mathrm{d} s=\iint_{A}\left(\frac{\partial D}{\partial t}+J\right) \cdot \mathrm{d} A, \\
& \iint_{\partial V} B \cdot \mathrm{d} A=0 \\
& \iint_{\partial V}\left(\frac{\partial D}{\partial t}+\boldsymbol{J}\right) \cdot \mathrm{d} \boldsymbol{A}=\mathbf{0},
\end{aligned}
$$

with the following relations:

$$
D=\epsilon E, \quad B=\mu H, \quad J=\kappa E+\rho v .
$$

A grid $\mathrm{G}$ is defined in the orthogonal coordinate system $r=r(u, v, w)$ as

$$
\begin{aligned}
\mathrm{G}= & \left\{\left(u_{i}, v_{j}, w_{k}\right) ; u_{1}<u_{i}<u_{I}, i=2, \ldots, I-1 ; v_{1}<v_{j}<v_{J}, j=2, \ldots, J-1 ;\right. \\
& \left.w_{1}<w_{k}<w_{K}, k=2, \ldots, K-1\right\} .
\end{aligned}
$$

All nodes of $\boldsymbol{G}$ are numbered linearly by

$$
\begin{aligned}
& n=1+(i-1) M_{u}+(j-1) M_{v}+(k-1) M_{w}, \\
& n=1, \ldots, N=I J K .
\end{aligned}
$$

Usually one uses $M_{u}=1, M_{v}=I, M_{w}=I J$ or any permutation of these three assignments. Figure 1 shows such a three-dimensional grid.

The functions which describe the field distribution in the volume under consideration are replaced by discrete field values in each cell of the grid. Thus one has to allocate components to grid nodes, lines, areas or elementary volumes. We will not choose the obvious allocation of calculating all six field components at the grid nodes. Such an allocation would cause serious problems at surfaces of materials where some of the field components are not continuous. Instead, the components of $E$ are placed at the mid-points of the edges of the grid cells and the components of $B$ at the centre of each face, as shown in fig. 2, extending the Yee Algorithm [1] to more general field problems [2-4].

This kind of allocation has the distinct advantage that the transition from one cell to the next involves only continuous components, tangential $\boldsymbol{E}$ fields and perpendicular $\boldsymbol{B}$ fields (see fig. 3); thus Maxwell's equations are always satisfied, in this respect, even when different material fillings are involved. We now apply a first-order integration formula in order to approximate the left hand side 


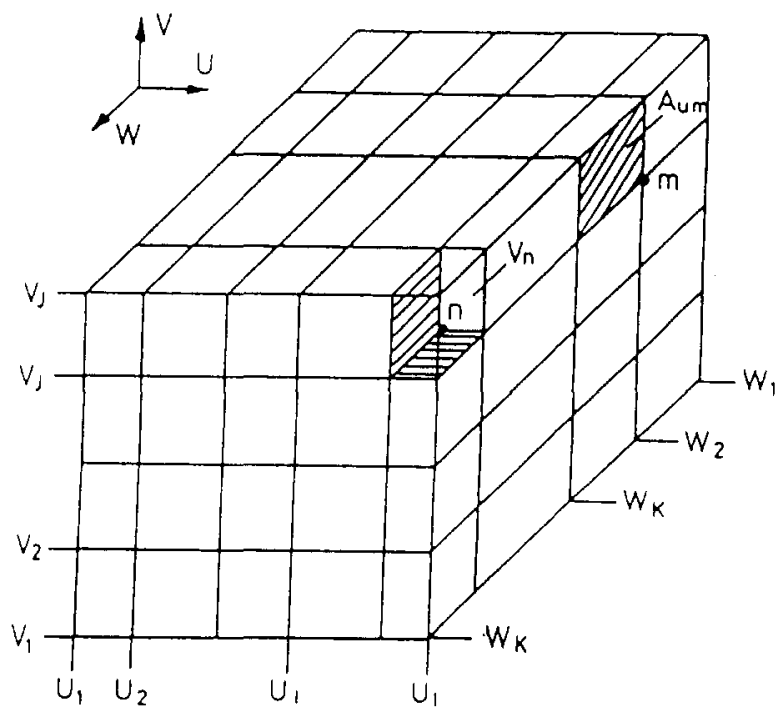

Fig. 1. Three-dimensional grid in the orthogonal not necessarily Cartesian system $(u, v, w)$.

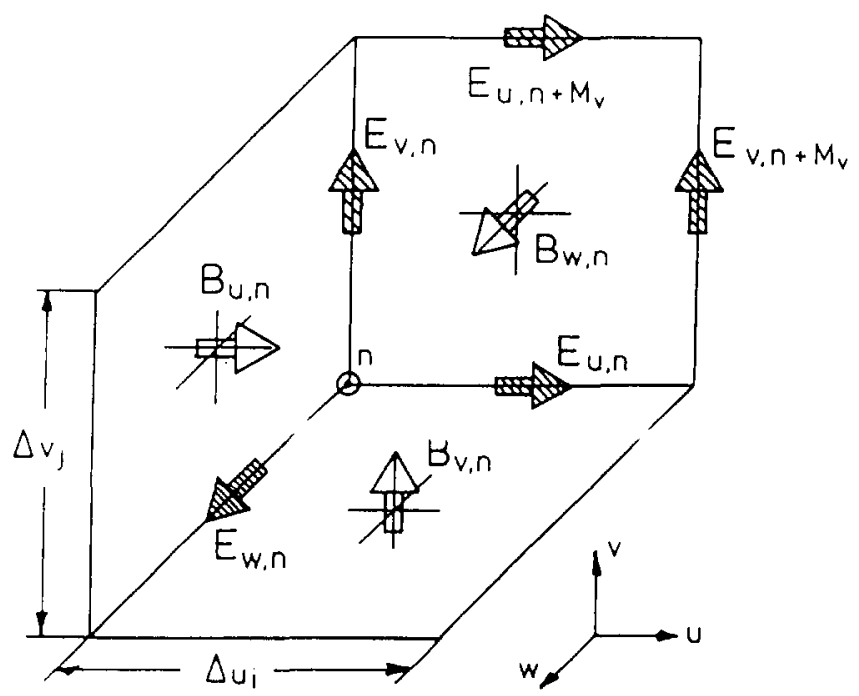

Fig. 2. Allocation of unknown field components in the grid $\mathrm{G}$ showing the indexing.

of eq. (1) and obtain the following algebraic expression replacing the integral along the border of an elementary cell:

$$
\begin{aligned}
\oint_{\partial \boldsymbol{A}_{w, n}} \boldsymbol{E} \cdot \mathrm{d} \boldsymbol{s}= & +\left(u_{i+1}-u_{i}\right) E_{u, n}+\left(v_{j+1}-v_{j}\right) E_{v, n+M_{u}}-\left(u_{i+1}-u_{i}\right) E_{u, n+M_{v}} \\
& -\left(v_{j+1}-v_{j}\right) E_{v, n}+\mathcal{O}\left(\left(u_{i+1}-u_{i}\right)^{2}\right)+\mathcal{O}\left(\left(v_{j+1}-v_{j}\right)^{2}\right) .
\end{aligned}
$$




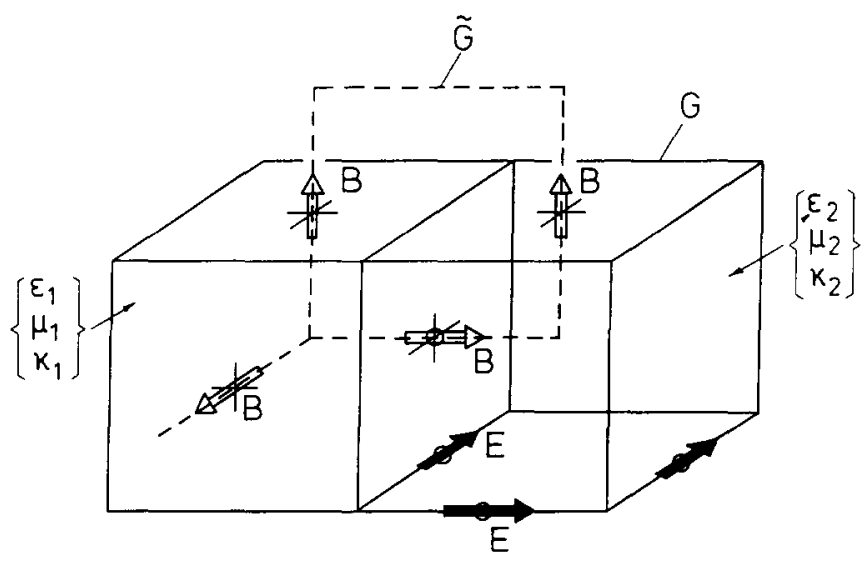

Fig. 3. Two neighbouring mesh cells showing the allocation of the electric and magnetic field components in $G$ and $\widetilde{G}$.

For the right hand side of that equation, we obtain (again by the lowest-order integration formula):

$$
\iint_{A_{w, n}} \frac{\partial \mathbf{B}}{\partial t} \cdot \mathrm{d} \mathbf{A}=\dot{\mathbf{B}}_{w, n}\left(u_{i+1}-u_{i}\right)\left(v_{j+1}-v_{j}\right)+\mathcal{O}\left(\left[\left(u_{i+1}-u_{i}\right)\left(v_{j+1}-v_{j}\right)\right]^{2}\right) .
$$

Equating the two expressions yields a discrete replacement for the first Maxwell equation on each surface of the grid cells and thus on every area composed of mesh cell surfaces.

In order to describe all these equations for all surfaces we introduce a basic discretization matrix with only two bands and with elements taking only the values $0,+1$ or -1 :

$$
\mathbf{P}_{u}:=\left(\mathbf{P}_{u}\right)_{m, p}= \begin{cases}-1, & p=m \\ +1, & p=m+M_{u}, \quad m, p=1, \ldots, N \\ 0, & \text { otherwise }\end{cases}
$$

Out of such simple matrices we combine the matrix $\mathbf{C}$ that replaces the contour integral operator in eq. (1) for all mesh points:

$$
\mathbf{C}=\left(\begin{array}{ccc}
\mathbf{0} & -\mathbf{P}_{w} & \mathbf{P}_{v} \\
\mathbf{P}_{w} & \mathbf{0} & -\mathbf{P}_{u} \\
-\mathbf{P}_{v} & \mathbf{P}_{u} & \mathbf{0}
\end{array}\right)
$$

All unknown components of the electric field $E$ are put into a vector of dimension $3 N=3(I J K)$ :

$$
e=\left(E_{u, 1}, E_{u, 2}, \ldots, E_{w, N}\right)^{\mathrm{t}}
$$

and we replace $D$ by $\boldsymbol{d}, \boldsymbol{B}$ by $\boldsymbol{b}, \boldsymbol{H}$ by $\boldsymbol{h}$ and $\mathbf{J}$ by $\boldsymbol{j}$, respectively. The topological part of the contour integral (curl) is represented by the matrix $\mathbf{C}$. The actual length of the integration path is put into a diagonal matrix:

$$
\mathbf{D}_{s}=\operatorname{Diag}\left(\Delta u_{1}, \ldots, \Delta u_{N}, \Delta v_{1}, \ldots, \Delta v_{N}, \Delta w_{1}, \ldots, \Delta w_{N}\right)
$$




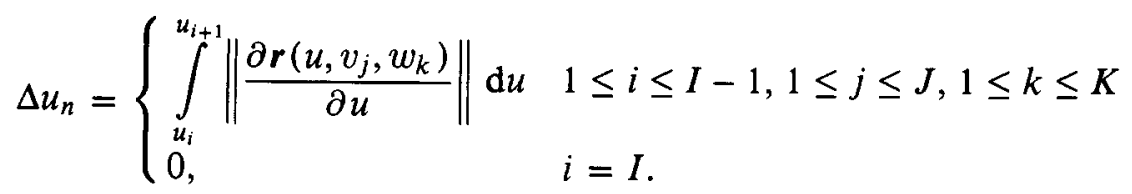

$\Delta v_{n}$ and $\Delta w_{n}$ are defined accordingly. The surfaces of the grid cells compose the diagonal matrix:

$$
\begin{aligned}
& \mathbf{D}_{A}=\operatorname{Diag}\left(A_{u, 1}, \ldots A_{u, N}, A_{v, 1}, \ldots, A_{v, N}, A_{w, 1}, \ldots, A_{w, N}\right), \\
& A_{u, n}=\Delta v_{n} \Delta w_{n}, \quad A_{v, n}=\Delta w_{n} \Delta u_{n}, \quad A_{w, n}=\Delta u_{n} \Delta v_{n} .
\end{aligned}
$$

Finally we can give a matrix equation using all these definitions that replaces the first Maxwell equation on all cell surfaces:

$$
\mathrm{CD}_{s} e=-\mathrm{D}_{A} \dot{b}
$$

The special allocation of field components in the grid generates a second, dual grid $\widetilde{G}$ in which the magnetic flux densities are allocated as are the electric field components in the original grid $\mathrm{G}$, see fig. 3.

The dual grid is defined by

$$
\begin{aligned}
\widetilde{\mathrm{G}}= & \left\{\left(\tilde{u}_{i}, \widetilde{v}_{j}, \widetilde{w}_{k}\right): \tilde{u}_{1}<\tilde{u}_{i}<\tilde{u}_{I}, i=2, \ldots, I-1 ; \widetilde{v}_{1}<\widetilde{v}_{j}<\widetilde{v}_{J}, j=2, \ldots, J-1 ;\right. \\
& \left.\widetilde{w}_{1}<\widetilde{w}_{k}<\widetilde{w}_{K}, k=2, \ldots, K-1\right\}, \\
\tilde{u}_{i}= & \left(u_{i+1}+u_{i}\right) / 2, \quad 1 \leq i \leq I-1, \widetilde{u}_{I}=0 \\
\widetilde{v}_{j}= & \left(v_{j+1}+v_{j}\right) / 2, \quad 1 \leq j \leq J-1, \tilde{v}_{J}=0 \\
\tilde{w}_{k}= & \left(w_{k+1}+w_{k}\right) / 2, \quad 1 \leq k \leq K-1, \widetilde{w}_{K}=0 .
\end{aligned}
$$

We can also define matrices that hold the mesh step sizes and cell areas as for the original grid G:

$$
\begin{aligned}
& \widetilde{\mathbf{D}}_{s}=\operatorname{Diag}\left(\widetilde{\Delta} u_{1}, \ldots, \widetilde{\Delta} u_{N}, \widetilde{\Delta} v_{1}, \ldots, \widetilde{\Delta} v_{N}, \widetilde{\Delta} w_{1}, \ldots, \widetilde{\Delta} w_{N}\right), \\
& \widetilde{\Delta} u_{n}= \begin{cases}\left(\Delta u_{n}+\Delta u_{n-M_{u}}\right) / 2, & 2 \leq i \leq I-1, \\
\Delta u_{n} / 2, & i=1, \\
\Delta u_{n-M_{u}} / 2, & i=I,\end{cases} \\
& \widetilde{\mathbf{D}}_{A}=\operatorname{Diag}\left(\tilde{A}_{u, 1}, \ldots, \tilde{A}_{u, N}, \tilde{A_{v, 1}}, \ldots, \widetilde{A_{v, N}}, \tilde{A}_{w, 1}, \ldots, \widetilde{A}_{w, N}\right) \text {, } \\
& \widetilde{A_{u, n}}=\widetilde{\Delta} v_{n} \widetilde{\Delta} w_{n}, \quad \widetilde{A_{v, n}}=\widetilde{\Delta} w_{n} \widetilde{\Delta} u_{n}, \quad \tilde{A_{w, n}}=\widetilde{\Delta} u_{n} \widetilde{\Delta} v_{n} .
\end{aligned}
$$

When solving the second Maxwell equation in a way similar to the first one, we have to face the fact that the magnetic field $\boldsymbol{H}$ is not defined and thus has to be calculated from the flux density piecewise along the integration path. Also, the integration over the electric flux density is no longer a simple product of an area with a component but the sum over four parts on each of which the flux has to be determined from the defined $E$ and the material constants. Finally we obtain:

$$
\widetilde{\mathbf{C}} \widetilde{\mathbf{D}}_{s} \mathbf{D}_{\mu}^{-1} \boldsymbol{b}=\widetilde{\mathbf{D}}_{A}(\dot{\boldsymbol{d}}+\dot{j}) \text {. }
$$

Note that the contour integral operator on the dual grid, $\widetilde{\mathbf{C}}$, (curl) is related to $\mathbf{C}$ simply by 


$$
\widetilde{\mathbf{C}}=\mathbf{C}^{\mathbf{t}}
$$

The diagonal matrices which take care of the piecewise different values for the material constants are given as

$$
\begin{aligned}
&\left(\mathbf{D}_{\mu}\right)_{n}=\tilde{\Delta} u_{n} /\left(\frac{\Delta u_{n-M_{u}}}{2 \mu_{u, n-M_{u}}}+\frac{\Delta u_{n}}{2 \mu_{u, n}}\right) \\
&\left(\widetilde{\mathbf{D}}_{\epsilon}\right)_{2 N+n}=\left(A_{w, n-M_{u}} \epsilon_{w, n-M_{u}}+A_{w, n-M_{v}} \epsilon_{w, n-M_{v}}\right. \\
&\left.+A_{w, n} \epsilon_{w, n}+A_{w, n-M_{u}-M_{v}} \epsilon_{w, n-M_{u}-M_{v}}\right) /\left(4 \widetilde{A}_{w, n}\right) \\
&\left(\widetilde{\mathbf{D}}_{\kappa}\right)_{2 N+n}=\left(A_{w, n-M_{u}} \kappa_{w, n-M_{u}}+A_{w, n-M_{v}} \kappa_{w, n-M_{v}}\right. \\
&\left.+A_{w, n} \kappa_{w, n}+A_{w, n-M_{u}-M_{v}} \kappa_{w, n-M_{u}-M_{v}}\right) /\left(4 \tilde{A}_{w, n}\right)
\end{aligned}
$$

while $\mu, \epsilon$ and $\kappa$ may have different properties in the directions $u, v$ and $w$. Although not originally defined in the grid, one may relate the field strength, flux density and current density and formally define a magnetic field and an electric flux density by

$$
\begin{array}{rlrl}
\boldsymbol{b} & =\mathbf{D}_{\mu} \boldsymbol{h} & & (\boldsymbol{B}=\mu \boldsymbol{H}), \\
\boldsymbol{d} & =\widetilde{\mathbf{D}}_{\epsilon} \boldsymbol{e} & & (\boldsymbol{D}=\epsilon E), \\
\boldsymbol{j} & =\widetilde{\mathbf{D}}_{\kappa} \boldsymbol{e}+\mathbf{D}_{\rho} \boldsymbol{v} & (\boldsymbol{J}=\kappa \boldsymbol{E}+\rho \boldsymbol{v}) .
\end{array}
$$

In order to complete the transformation of Maxwell's equations to the grid space, we approximate the divergence equation by integrating $B$ over surfaces of each mesh cell of $G$. We can then write this equation by defining the discrete div operator on $G$ as

$$
\mathbf{S}=\left(\boldsymbol{P}_{u}, \boldsymbol{P}_{v}, \boldsymbol{P}_{w}\right)
$$

and obtain

$$
\mathbf{S D}_{A} b=\mathbf{0}
$$

This equation allocates the (nonexistent) magnetic charges to nodes of $\widetilde{G}$. The corresponding matrix on the dual grid $\widetilde{G}$ is simply given by

$$
\widetilde{\mathbf{S}}=\left(\boldsymbol{P}_{u}^{\mathbf{t}}, \boldsymbol{P}_{v}^{\mathrm{t}}, \boldsymbol{P}_{w}^{\mathrm{t}}\right)
$$

So the continuity equation in the grid space reads

$$
\widetilde{\mathbf{S}} \widetilde{\mathbf{D}}_{A}(\dot{d}+j)=\mathbf{0}
$$

Thus electric charges are defined on nodes of the original grid $\mathrm{G}$. 


\section{Properties of the grid equations}

One of the outstanding properties of Maxwell's grid equations is that properties of analytical solutions have their exact analogues in the grid space. The analytical identity div curl $\equiv 0$ reads in the grid space as

$$
\operatorname{div} \operatorname{curl} \equiv 0 \Leftrightarrow \tilde{\mathbf{S C}} \equiv \mathbf{S C} \equiv \mathbf{0}
$$

This identity in grid space can easily be proved by showing that:

$$
\mathrm{SC}=(\mathbf{0}, \boldsymbol{0}, \mathbf{0})=\left(\boldsymbol{P}_{v} \boldsymbol{P}_{w}-\boldsymbol{P}_{w} \boldsymbol{P}_{v}, \boldsymbol{P}_{w} \boldsymbol{P}_{u}-\boldsymbol{P}_{u} \boldsymbol{P}_{w}, \boldsymbol{P}_{u} \boldsymbol{P}_{v}-\boldsymbol{P}_{v} \boldsymbol{P}_{u}\right)
$$

Thus the proof reduces to the equivalence of interchanged partial differentiation:

$$
\boldsymbol{P}_{u} \boldsymbol{P}_{v}=\boldsymbol{P}_{v} \boldsymbol{P}_{u} \Leftrightarrow\left(\frac{\partial}{\partial u} \frac{\partial}{\partial v}=\frac{\partial}{\partial v} \frac{\partial}{\partial u}\right)
$$

This matrix identity can easily be verified. The analytical property that scalar potential fields are curl free is also found in the grid space as

$$
\operatorname{curl} \operatorname{grad} \equiv 0 \Leftrightarrow \mathbf{C}^{\mathbf{t}} \mathbf{S}^{\mathrm{t}} \equiv \widetilde{\mathbf{C}} \widetilde{\mathbf{S}}^{\mathrm{t}} \equiv \mathbf{0} .
$$

The proof of this identity is found by simply transposing the identity (div curl $\equiv 0$ ) eq. (45). Thus the source-freeness of the "curly" field is in that sense equivalent to the fact that the scalar gradient field is irrotational.

These properties of the Maxwell grid equations not only offer a unique tool to test numerical results for their physical correctness but also avoid the occurrence of incorrect solutions in the calculation of three-dimensional eigenmodes in resonators, or at least their identification [3]. This is quite important since numerical errors in the matrix algorithms may occur and cannot generally be detected. In the field of accelerator physics, one often investigates unknown phenomena by using such codes, so that an incorrect solution could lead to physical misinterpretations.

\section{The Maxwell grid equations}

Without specifying anything about the shape of materials nor the time dependence of the fields, we have obtained a set of matrix equations that approximate Maxwell's equations on a double grid $G, \widetilde{G}$ $\left(\mathbb{R}^{3}\right.$ and $\mathbb{R}^{3 N}$ represent the physical spaces and $\mathbb{R}_{+}$the time):

$$
\begin{array}{lll}
\text { Real space } \mathbb{R}^{3} \otimes \mathbb{R}_{+} & \Leftrightarrow & \text { Grid space } \mathbb{R}^{3 N} \otimes \mathbb{R}_{+}, \\
\oint_{\partial A} E \cdot \mathrm{d} s=-\iint_{A} \frac{\partial B}{\partial t} \cdot \mathrm{d} A & \Leftrightarrow & \mathbf{C D}_{s} e=-\mathbf{D}_{A} \dot{b} \\
\oint_{\partial A} \boldsymbol{H} \cdot \mathrm{d} s=\iint\left(\frac{\partial D}{\partial t}+J\right) \cdot \mathrm{d} \boldsymbol{A} & \Leftrightarrow & \widetilde{\mathbf{C}} \widetilde{\mathbf{D}}_{s} \boldsymbol{h}=\widetilde{\mathbf{D}}_{A} \dot{d}+\boldsymbol{j}
\end{array}
$$




$$
\begin{array}{ll}
\iint_{V} B \cdot \mathrm{d} \boldsymbol{A}=\mathbf{0} & \Leftrightarrow \mathbf{S D}_{A} \boldsymbol{b}=\mathbf{0}, \\
\iint_{V}\left(\frac{\partial \boldsymbol{D}}{\partial t}+\boldsymbol{J}\right) \cdot \mathrm{d} \boldsymbol{A}=0 & \Leftrightarrow \tilde{\mathbf{S}} \widetilde{\mathbf{D}}_{A}(\dot{\boldsymbol{d}}+\boldsymbol{j})=\mathbf{0}, \\
\boldsymbol{D}=\epsilon \boldsymbol{E} & \Leftrightarrow \boldsymbol{d}=\widetilde{\mathbf{D}}_{\epsilon} \boldsymbol{e}, \\
\boldsymbol{B}=\mu \boldsymbol{H} & \Leftrightarrow \boldsymbol{b}=\mathbf{D}_{\mu} \boldsymbol{h}, \\
\boldsymbol{J}=\kappa \boldsymbol{E}+\rho \boldsymbol{v} & \Leftrightarrow \boldsymbol{j}=\tilde{\mathbf{D}}_{\kappa} \boldsymbol{e}+\mathbf{D} \boldsymbol{\nu}, \\
\operatorname{div} \operatorname{curl} \equiv \mathbf{0} & \Leftrightarrow \mathbf{S C}=\widetilde{\mathbf{S}} \widetilde{\mathbf{C}}=\mathbf{0}, \\
\operatorname{curl} \operatorname{grad} \equiv \mathbf{0} & \Leftrightarrow \tilde{\mathbf{C}}^{\mathrm{t}} \widetilde{\mathbf{S}}^{\mathrm{t}}=\mathbf{C}^{\mathrm{t}} \mathbf{S}^{\mathrm{t}}=\mathbf{0} .
\end{array}
$$

\section{Special cases of Maxwell equations}

In order to solve these equations one has only to perform matrix manipulations and then solve the established matrix problem numerically.

\subsection{Static fields}

In the case of static fields it is in general not necessary to describe the problem by vectors. Gradients of a scalar potential are used to derive the Poisson equation:

$$
\begin{aligned}
& E=-\operatorname{grad} \phi_{E}, \\
& \operatorname{div}\left(\epsilon \operatorname{grad} \phi_{E}\right)=-\rho .
\end{aligned}
$$

Instead of numerically solving Maxwell's equation we define the electric potentials on nodes of $G$ as

$$
\boldsymbol{\Phi}_{e}=\left(\phi_{E, 1}, \phi_{E, 2}, \phi_{E, 3}, \ldots, \phi_{E, N}\right)^{t}
$$

and derive the corresponding equations only by matrix manipulation:

$$
\begin{aligned}
& \boldsymbol{e}=-\widetilde{\mathbf{S}}^{t} \mathbf{D}_{s}^{-1} \boldsymbol{\Phi}_{e}, \\
& \widetilde{\mathbf{S}} \tilde{\mathbf{D}}_{\epsilon} \widetilde{\mathbf{D}}_{A} \mathbf{D}_{s}^{-1} \widetilde{\mathbf{S}}^{\mathrm{t}} \boldsymbol{\Phi}_{e}=-\boldsymbol{q} .
\end{aligned}
$$

The latter equation is of the order $N$ and is the "Grid Potential Equation". The right hand side contains all charges on the nodes in the vector $q$ of dimension $N$.

For the magnetostatic field the procedure is similar except that the "curly" part of the magnetic field has to be taken out in order to allow the use of a scalar potential:

$$
\begin{aligned}
& \boldsymbol{H}=\boldsymbol{H}_{\mathrm{c}}-\operatorname{grad} \phi_{H}, \\
& \operatorname{curl} \boldsymbol{H}_{\mathrm{c}}=\boldsymbol{J} \\
& \operatorname{div}\left(\mu \operatorname{grad} \phi_{H}\right)=\operatorname{div}\left(\mu \boldsymbol{H}_{\mathrm{c}}\right) .
\end{aligned}
$$


Here the right hand side is given by "magnetic charges" which result from the curly field but have otherwise no physical meaning. Equation (65) is equivalent to (59) where $\mu, \boldsymbol{H}$ and $\operatorname{div}\left(\mu \boldsymbol{H}_{\mathrm{c}}\right)$ correspond to $\epsilon, E$ and $\rho$. One obtains the same matrix equations by allocating $\boldsymbol{H}$ components on the grid and calculating $\widetilde{\mathbf{D}}_{\mu}$ similarly to $\widetilde{\mathbf{D}}_{\epsilon}$ :

$$
\begin{aligned}
& \boldsymbol{h}=\boldsymbol{h}_{\mathrm{c}}-\widetilde{\mathbf{S}}^{\mathrm{t}} \mathbf{D}_{s}^{-1} \boldsymbol{\Phi}_{h}, \\
& \mathbf{C D}_{s} \boldsymbol{h}_{\mathrm{c}}=\widetilde{\mathbf{D}}_{A} \boldsymbol{j}, \\
& \widetilde{\mathbf{S}} \widetilde{\mathbf{D}}_{\mu} \widetilde{\mathbf{D}}_{A} \mathbf{D}_{s}^{-1} \widetilde{\mathbf{S}}^{\mathrm{t}} \boldsymbol{\Phi}_{h}=\widetilde{\mathbf{S}} \widetilde{\mathbf{D}}_{\mu} \widetilde{\mathbf{D}}_{A} \boldsymbol{h}_{c} .
\end{aligned}
$$

This allows the use of the same iterative algorithm for solving electro- and magnetostatic problems.

\subsection{Time harmonic and resonant fields}

The electric fields simultaneously have to obey the following two equations, that can be directly derived from Maxwell's equations

$$
\begin{aligned}
& \operatorname{curl} \frac{1}{\mu} \operatorname{curl} E=\omega^{2} \epsilon E-i \omega \kappa E-i \omega J, \\
& \operatorname{div} \epsilon E=\frac{1}{i \omega}(\operatorname{div} \kappa E+\operatorname{div} J) .
\end{aligned}
$$

The numerical formulation can be derived again by matrix manipulation:

$$
\begin{aligned}
& \left(\widetilde{\mathbf{C}} \widetilde{\mathbf{D}}_{s} \mathbf{D}_{\mu}^{-1} \mathbf{D}_{A}^{-1} \mathbf{C D}_{s}+\mathrm{i} \omega \widetilde{\mathbf{D}}_{A} \widetilde{\mathbf{D}}_{\kappa}-\omega^{2} \widetilde{\mathbf{D}}_{A} \widetilde{\mathbf{D}}_{\epsilon}\right) \boldsymbol{e}=-\mathrm{i} \omega \widetilde{\mathbf{D}}_{A} \mathbf{D}_{\rho} \boldsymbol{v}, \\
& \widetilde{\mathbf{S}} \widetilde{\mathbf{D}}_{A} \widetilde{\mathbf{D}}_{\epsilon} \boldsymbol{e}=\frac{1}{i \omega}\left(\tilde{\mathbf{S}} \widetilde{\mathbf{D}}_{A} \widetilde{\mathbf{D}}_{\kappa} \boldsymbol{e}+\widetilde{\mathbf{S}} \widetilde{\mathbf{D}}_{A} \mathbf{D}_{\rho} \boldsymbol{v}\right) .
\end{aligned}
$$

Equation (71) represents a $3 N \times 3 N$ equation system, which can usually be solved unless the stimulation frequency $\omega$ corresponds to a system resonance frequency. The resulting matrix, however, has a multiply vanishing eigenvalue for $\omega=0$ as the existence of static solutions cannot be excluded by curl operations only. Positive frequencies yield eigenvalues with a negative real part, which create problems for many algorithms for the solution of equation systems. That is why the combination of eq. (71) with derivations of eq. (72) seems to be advantageous so that a better distribution of eigenvalues can be achieved. A variety of combinations is possible; e.g. with any diagonal matrix $\mathbf{D}_{V}$ which vanishes for every point where eq. (72) does not, the following $3 N \times 3 N$ system can be derived:

$$
\widetilde{\mathbf{D}}_{s}^{\mathrm{t}} \mathbf{D}_{V} \widetilde{\mathbf{S}} \widetilde{\mathbf{D}}_{A} \widetilde{\mathbf{D}}_{\epsilon} \boldsymbol{e}=\mathbf{0} .
$$

Equation (72) vanishes for all grid-points which are not on a surface where material properties change and for which the current density is source free. In order to convert the algorithm to a symmetric form we must perform a diagonal transformation, which physically replaces the field strength by the root of the local energy density (except from a scalar factor):

$$
\begin{aligned}
& \boldsymbol{e}^{\prime}=\left(\widetilde{\mathbf{D}}_{\epsilon} \widetilde{\mathbf{D}}_{A} \mathbf{D}_{s}\right)^{1 / 2} \boldsymbol{e} \\
& \left((\widetilde{\mathbf{D}} \widetilde{\mathbf{C}} \mathbf{D})(\widetilde{\mathbf{D}} \widetilde{\mathbf{C}} \mathbf{D})^{t}+i \omega \widetilde{\mathbf{D}}_{\kappa} \widetilde{\mathbf{D}}_{\epsilon}^{-1}-\omega^{2} \mathbf{I}\right) \boldsymbol{e}^{\prime}=-i \omega \widetilde{\mathbf{D}} \widetilde{\mathbf{D}}_{A} \mathbf{D}_{\rho} \boldsymbol{\nu},
\end{aligned}
$$




$$
\widetilde{\mathbf{D}}^{-1} \widetilde{\mathbf{D}}_{s}^{\mathrm{t}} \mathbf{D}_{V} \widetilde{\mathbf{S}} \widetilde{\mathbf{D}}^{-1} \boldsymbol{e}^{\prime}=\mathbf{0},
$$

with the matrices

$$
\begin{aligned}
& \mathbf{D}=\left(\mathbf{D}_{A}^{-1} \widetilde{\mathbf{D}}_{s} \mathbf{D}_{\mu}^{-1}\right)^{1 / 2}, \\
& \widetilde{\mathbf{D}}=\left(\widetilde{\mathbf{D}}_{A}^{-1} \mathbf{D}_{s} \widetilde{\mathbf{D}}_{\epsilon}^{-1}\right)^{1 / 2} .
\end{aligned}
$$

Solving a combination of these linear systems for a given driving current distribution yields the field at all locations in G. Further specialisation to e.g. low-frequency eddy currents invokes mixing of scalar fields and vector fields. For a loss free medium with no driving current one obtains an eigenvalue equation, the eigenvalues of which are the resonant frequencies squared [3]:

$$
(\widetilde{\mathbf{D}} \widetilde{\mathbf{C}})(\widetilde{\mathbf{D}} \widetilde{\mathbf{C}} \mathbf{D})^{t} \boldsymbol{e}^{\prime}=\omega^{2} \boldsymbol{e}^{\prime}
$$

The above mentioned multiple eigenvalue at zero can be avoided by combining eq. (79) with (76). As the total number of eigenvalues remains at $3 N$, unphysical eigenvalues appear, which can be identified easily by resubstituting the eigenvectors in eqs. (79) and (76).

The analytical analogue to this combination of eqs. (75) or (79) with (76) is the equation curl curl $\boldsymbol{E}=\operatorname{grad} \operatorname{div} E-\nabla^{2} \boldsymbol{E}$ and $\operatorname{div} \boldsymbol{E}=0$ thus solving $\nabla^{2} \boldsymbol{E}$.

\subsection{Fields in the time domain}

For transient fields with a central time difference formula, step size $\delta t$, no currents and no losses, one obtains the Yee-algorithm [1]. In the presence of free moving charges the algorithm has more constraints to ensure charge conservation [5]. The upper index $n$ denotes the time in units of $\delta t$. These recursive formulae allow the easy calculation of transient fields of antennas, particle beams in accelerators [5] or wave propagation problems even in the lossy case:

$$
\begin{aligned}
& \boldsymbol{b}^{n+1}=\boldsymbol{b}^{n}-\Delta t \mathbf{D}_{A}^{-1} \mathbf{C D}_{s} \boldsymbol{e}^{n+0.5}, \\
& \boldsymbol{e}^{n+1.5}=\boldsymbol{D}_{a} \boldsymbol{e}^{n+0.5}+\left(\mathbf{I}-\mathbf{D}_{a}\right) \widetilde{\mathbf{D}}_{\kappa}^{-1} \widetilde{\mathbf{D}}_{A}^{-1} \widetilde{\mathbf{C}} \widetilde{\mathbf{D}}_{s} \mathbf{D}_{\mu}^{-1} \boldsymbol{b}^{n+1}-\left(\mathbf{I}-\mathbf{D}_{a}\right) \widetilde{\mathbf{D}}_{\kappa}^{-1} \boldsymbol{j}_{e}^{n+1},
\end{aligned}
$$

with

$$
\mathbf{D}_{a}=\exp \left(-\widetilde{\mathbf{D}}_{\epsilon}^{-1} \widetilde{\mathbf{D}}_{\kappa} \Delta t\right)
$$

The detailed setting up of the final matrix problem to be solved depends too much on the specific problem to be explained here for all possible cases. We refer to refs. $[3,4]$ and will demonstrate the wide applicability of this method by means of a few examples.

\section{Description of the MAFIA programs}

The MAFIA programs are a set of two- and three-dimensional computer codes based on the Maxwell Grid Equations. They were developed for use in the computer-aided design of particle accelerators and are now finding wider applications in other fields such as tomography, filters, integrated circuits and resonators. See table 1. 
Table 1

Description of the MAFIA programs.

\begin{tabular}{|c|c|c|c|}
\hline Module & Description & Special features & Geometry \\
\hline $\mathbf{M}$ & $\begin{array}{l}\text { mesh generator, translates the } \\
\text { physical problem into mesh data }\end{array}$ & many predefined shapes, interactive remeshing & $\begin{array}{l}(x, y, z), \quad(x, y) \\
(r, z)\end{array}$ \\
\hline $\mathbf{S}$ & $\begin{array}{l}\text { static solver, electro- and magne- } \\
\text { tostatic problems }\end{array}$ & $\begin{array}{l}\text { open boundary, nonlinear magnetic material } \\
\text { properties, advanced multigrid solver for very } \\
\text { large meshes }\end{array}$ & $(x, y, z),(r, z)$ \\
\hline $\mathbf{R}, \mathbf{E}$ & $\begin{array}{l}\text { matrix generator, eigenvalue sol- } \\
\text { ver for resonator and waveguide } \\
\text { problems }\end{array}$ & $\begin{array}{l}\text { periodic boundary conditions, resonance fre- } \\
\text { quencies (several hundred modes), waveguide } \\
\text { modes and propagation parameters }\end{array}$ & $\begin{array}{l}(x, y, z), \quad(x, y) \\
(r, z)\end{array}$ \\
\hline $\mathrm{T} 2, \mathrm{~T} 3$ & time domain solver & $\begin{array}{l}\text { losses, open boundary, stimulation by: initial } \\
\text { field, current, waveguide, incident waves; wake } \\
\text { potentials }\end{array}$ & $(r, z),(x, y, z)$ \\
\hline TS2, TS3 & $\begin{array}{l}\text { particle in cell code, calculates } \\
\text { equation of motion for free mov- } \\
\text { ing charges }\end{array}$ & $\begin{array}{l}\text { losses, stimulation by initial field, calculation } \\
\text { of trajectories, velocities, charge densities, etc. }\end{array}$ & $(x, y, z),(r, z)$ \\
\hline W3 & $\begin{array}{l}\text { eddy current solver, frequency } \\
\text { domain }\end{array}$ & losses, current stimulation & $(x, y, z)$ \\
\hline $\mathbf{P}$ & $\begin{array}{l}\text { post processor, displays results } \\
\text { and calculates secondary field } \\
\text { quantities }\end{array}$ & $\begin{array}{l}1,2 \text { and } 3 D \text { graphics of scalar and vector fields, } \\
\text { power loss integrals, field energy, far field pat- } \\
\text { tern, shunt impedance }\end{array}$ & $\begin{array}{l}(x, y, z), \quad(x, y) \\
(r, z)\end{array}$ \\
\hline
\end{tabular}

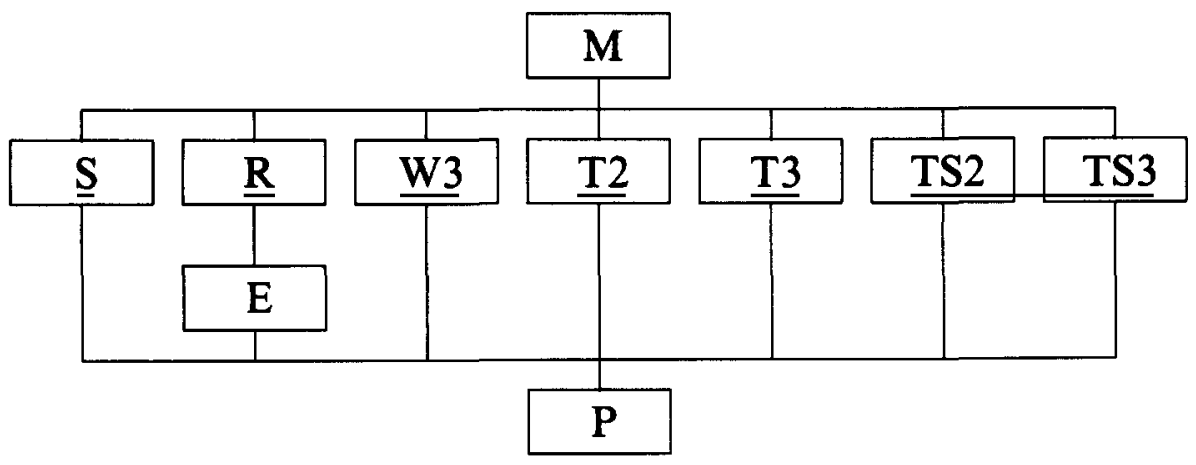

Fig. 4. The MAFIA system with its interrelationships.

The acronym MAFIA stands for the solution of MAxwell's equations by the Finite Integration Algorithm. Figure 4 shows the interconnections between the various programs ot the third release [6]. The second release of the MAFIA Programs, comprising M3, R3, E31, E32 and P3, has been distributed to over 120 installations worldwide including most countries of Europe, USA, USSR, China, Japan, India and Brazil. The programs have already proved their worth through comparison with theoretical calculations and by the successful design of accelerator components. The MAFIA codes are written in standard FORTRAN77 and currently run on IBM, CRAY, VAX, APOLLO, HP, SUN, CONVEX, AMDAHL, FUJITSU, HITACHI and STELLAR computers, among others. The distribution center for codes and userguides is the Technische Hochschule Darmstadt, for information contact Prof. Dr.Ing. Thomas Weiland. 

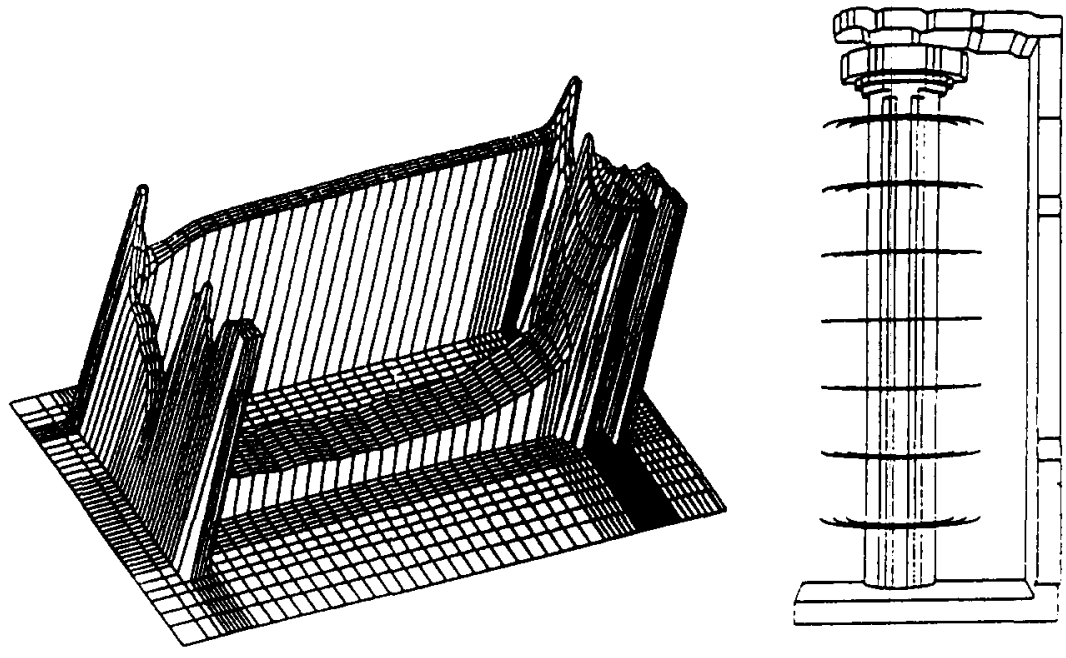

Fig. 5. Netplot of the field dependant $\mu$ in the symmetry plane of the relay.

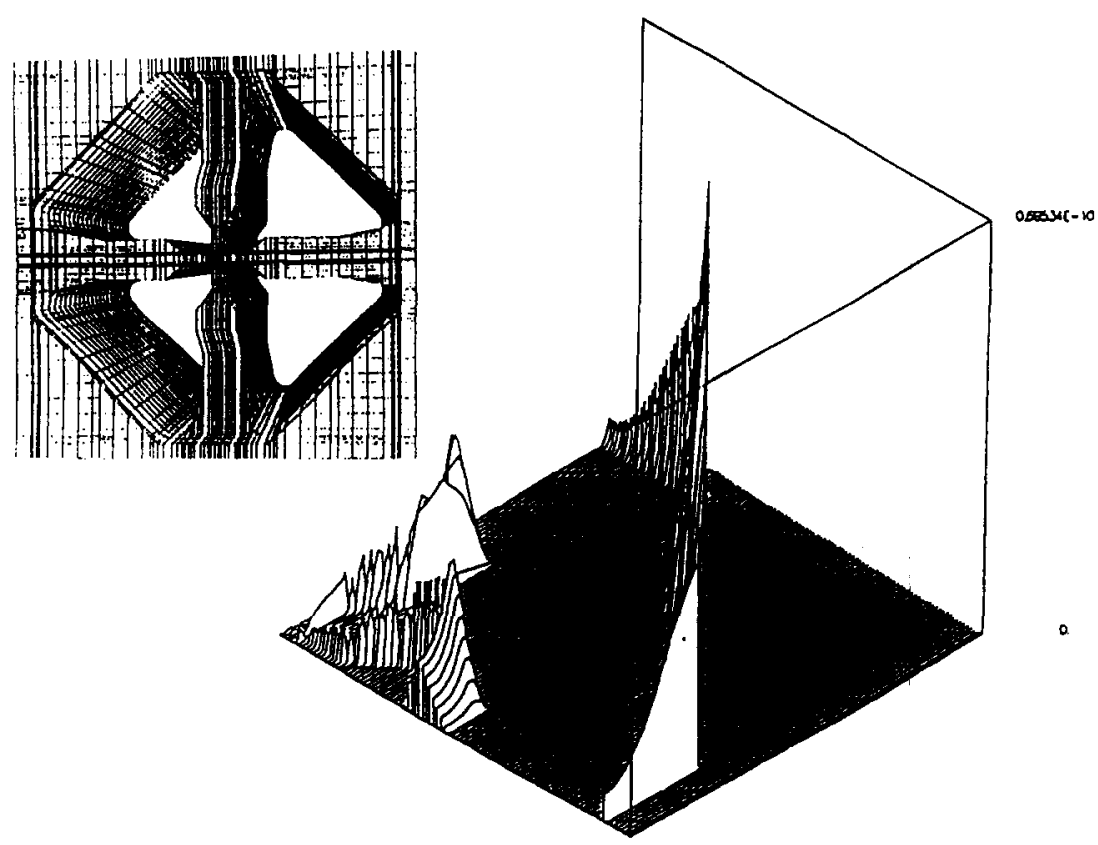

Fig. 6. The left of this figure shows a 3D perspective of the end region of the RFQ. Only one quarter of this structure needs to be calculated, due to existing symmetries. For this structure the power loss in a cut plane at the and of the structure is displayed. 


\section{Applications}

The applicability of this method and of the computer codes based on it is almost unlimited. In this short presentation we can only give a few typical applications from the area of electrical engineering and accelerator physics.

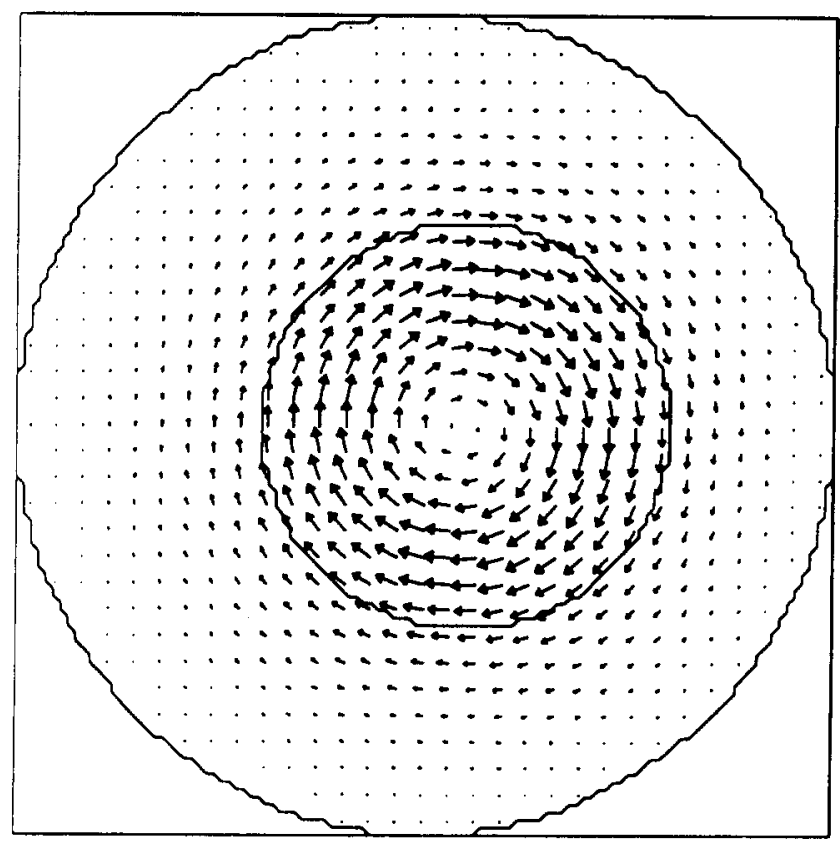

Fig. 7. Arrowplot of the electric field of the $E_{01}$-mode in a circular waveguide with an asymmetrically placed dielectric rod. (Geometry: $R=1 \mathrm{~m}, r / R=0.5$, offset $/ R=0.1, \epsilon_{R}=1, \epsilon_{r}=8, f=119.36 \mathrm{MHz}$ ).

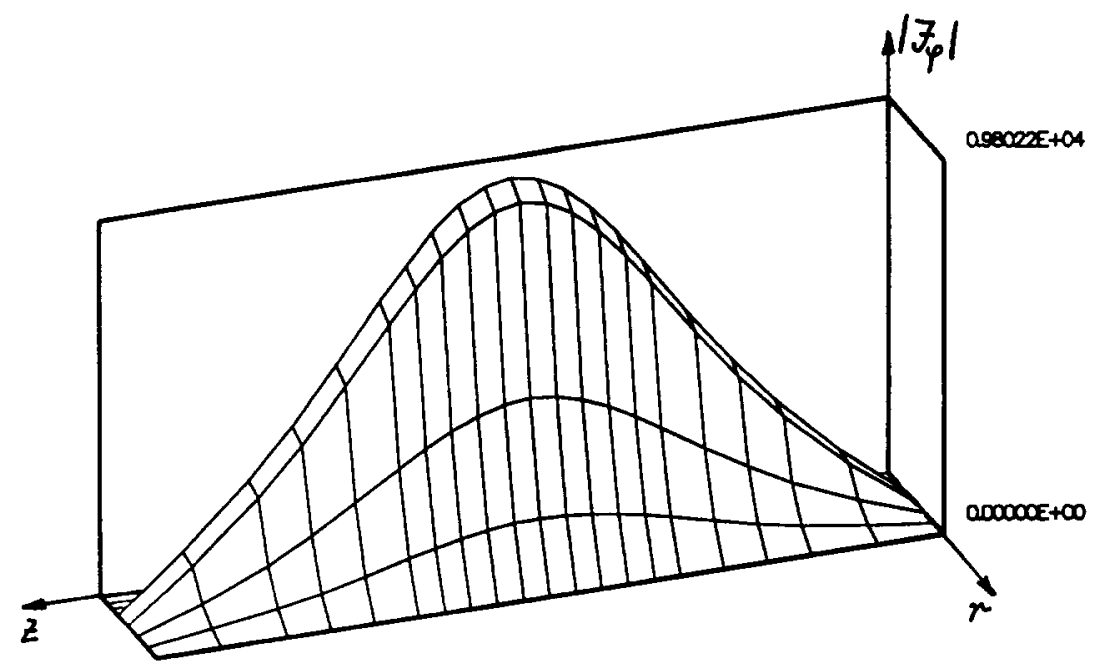

Fig. 8. Nondestructive testing of a tube by inducing an azimuthal eddy current by means of a single coil at the position $z=0$. 
The first example is an application of the MAFIA magneto- and electrostatic module S [8,9]. Relays are electric switching devices. The design goal for such a device might be to reduce its weight, the needed driving current or to minimize the force at the moving armature. This nonlinear magnetostatic problem has been calculated with our static module and also computed with the well known PROFI. The comparison has been performed for the achievable accuracy and the computational effort. The agreement in the results was reasonable. $S$ has the advantage that the use of open boundary conditions allows a description of this structure with less unknowns than is necessary when using standard Dirichlet or Neumann boundary conditions. Here a possible reduction in the number of unknowns of $10 \%$ reduced the needed CPU time by about $20 \%$. Figure 5 displays the relay and the $\mu$-distribution inside a cut through the central plane of the relay. One can clearly see the saturation effect at the bottom of the structure and inside the top armature, that indicate the necesity of redesigning the relay in order to obtain reasonable behaviour.

The next example is a radiofrequency quadrupole (RFQ) that has been calculated with the MAFIA frequency domain module $R / E$ [10]. The device might be tuned for a certain minimum eigenmode or in order to minimize the losses. Three-dimensional calculations are important especially for the end region of such a RFQ. A careful design using the MAFIA mesh genarator has been carried out, using about 75000 unknowns. The lowest eigenmode calculated, $344.78 \mathrm{MHz}$ is in good agreement with measurements and other calculations. Figure 6 displays the power loss. This loss is strongest on the

Table 2

Comparison of $\beta(1 / \mathrm{m})$ of ref. [7] with our method.

\begin{tabular}{lll}
\hline Mode & Solution $[7] \beta(1 / \mathrm{m})$ & Numeric solution $\beta(1 / \mathrm{m})$ \\
\hline 1 & 5.649 & 5.649 \\
2 & 5.648 & 5.641 \\
3 & 4.204 & 4.19 \\
4 & 3.079 & 3.082 \\
\hline
\end{tabular}

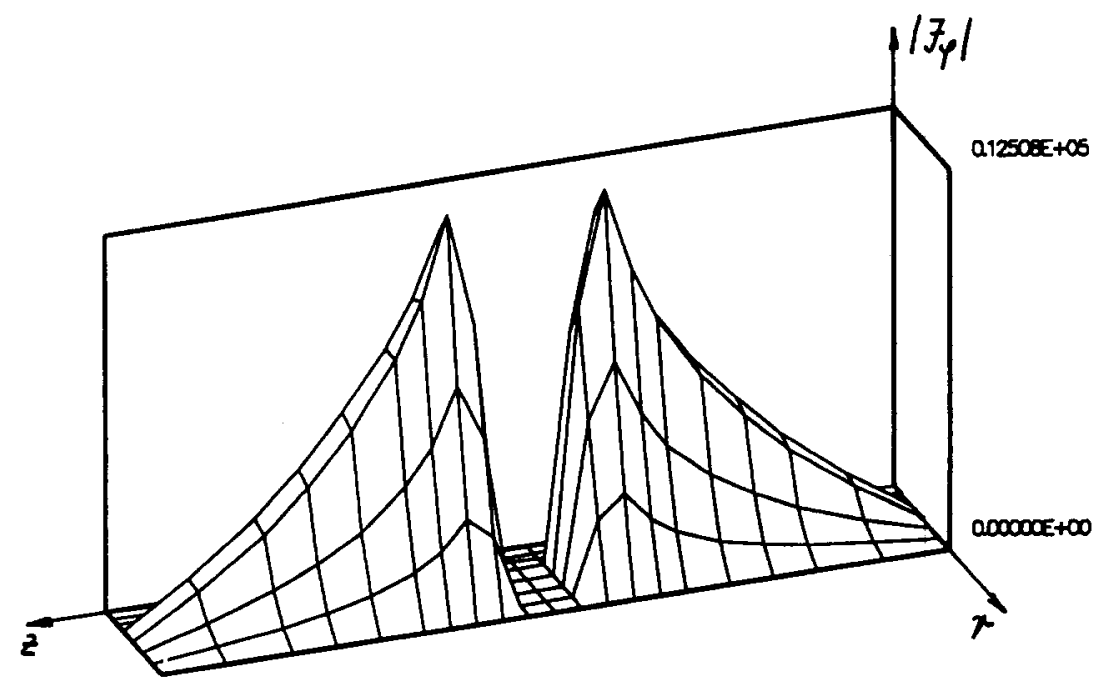

Fig. 9. Same tube and same stimulation as in the above figure but the tube has a radial hole at $z=0$. 


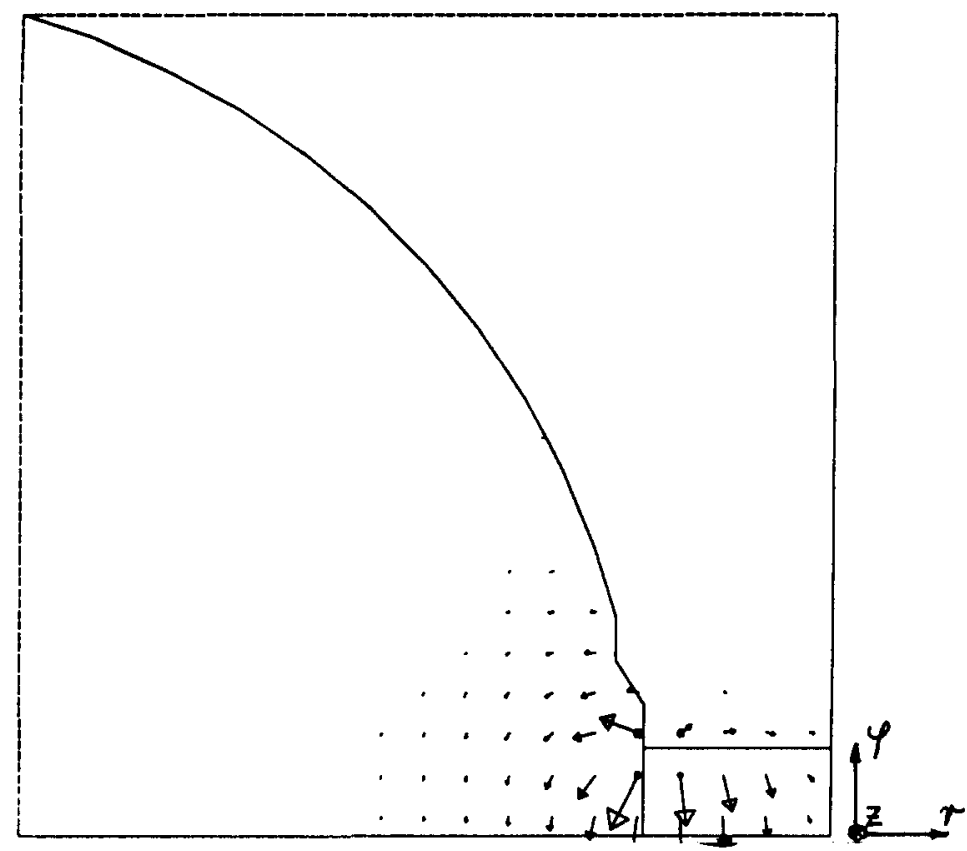

Fig. 10. Difference of the electric fields of the tube with and without the hole.

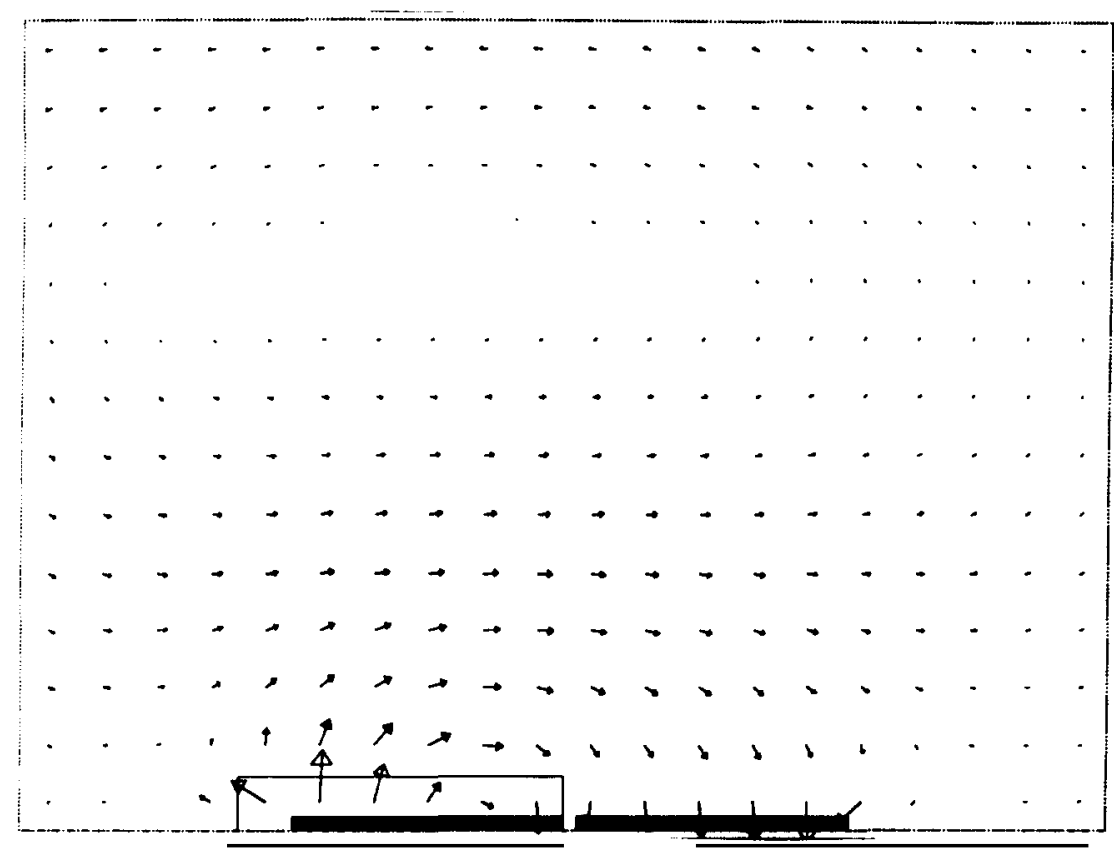

Fig. 11. Electric field of a $\lambda / 2$-dipole with an unsymmetric coating. Since the geometrical variation of the fields is small, only a mesh sized $\lambda \times 0.75 \lambda$ had to be computed. 
outside wall. The power loss can be calculated by the MAFIA-postprocessor from the field solutions. Different special approaches exist for the analysis of the eigenvalues and field components of dielectrically loaded waveguides. It is necessary to know these values to be able to estimate the influence of mechanical tolerances and to design filters and resonators. Table 2 compares semianalytical results for the phase constant $\beta$ of a calculation similar to [7] with the values achieved by our method. Figure 7 shows the electric vector components of the third mode $\left(E_{01}\right.$-mode).

A typical problem for nondestructive testing (NDT) is the detection of cracks and holes by means of eddy currents [11]. For example a tube can be tested by moving a coil through the tube and measuring its impedance. Material defects in the conducting tube are detected by the change in the impedance. In

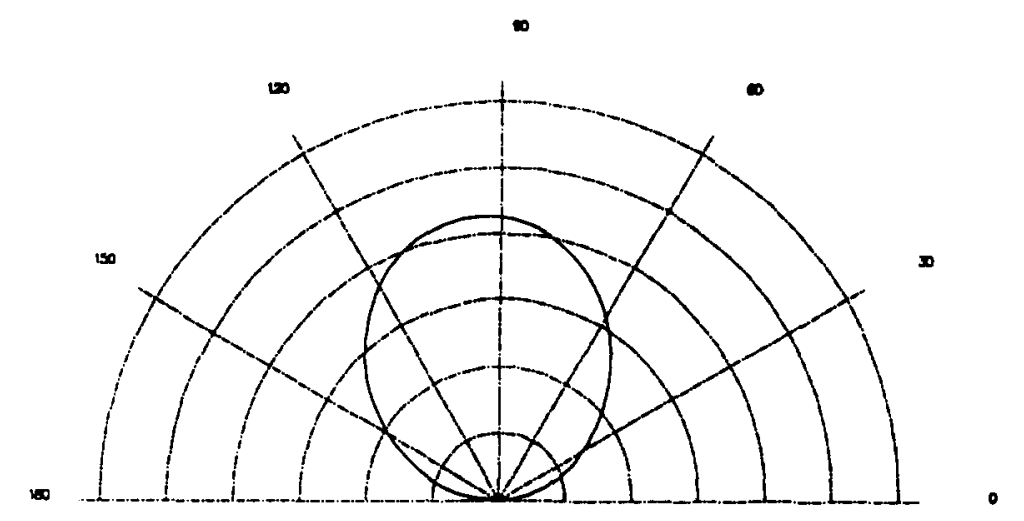

Fig. 12. Far field diagram for the field distribution as shown in the last figure. The maximum of the far field amplitude is shifted towards higher $\theta$ values.

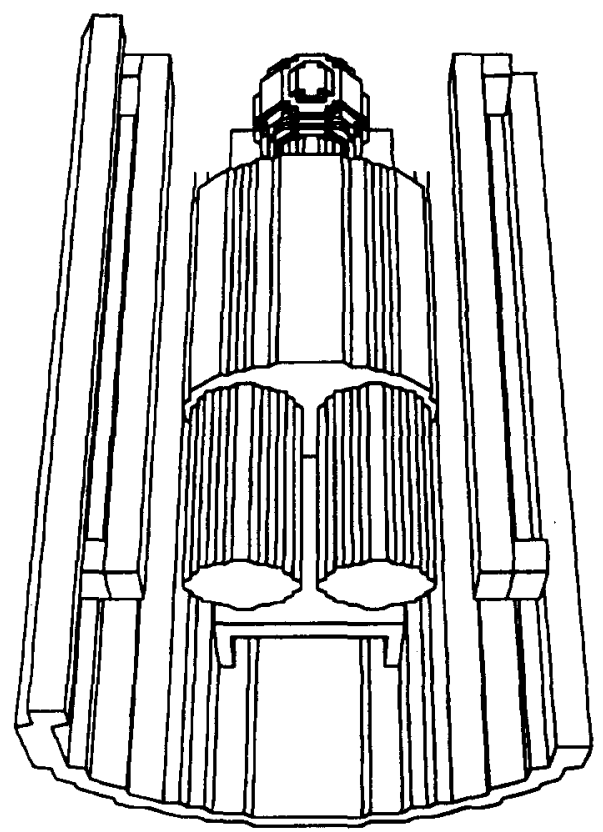

Fig. 13. Human test body in a three-dimensional nuclear resonance spectrometer. 
the following example the azimuthally oriented coil excites an azimuthal current density field, which is shown in fig. 8. The $z$-coordinate axis is coincident with the axis of the tube and the coil, the $x$ coordinate axis here corresponds to the radial coordinate. In a second calculation a hole in the tube wall was modelled. The resulting current density distribution in the plane normal to the hole is shown in fig. 9. The electric fields induce a voltage in the stimulation coil, the difference of the fields with and without the hole is plotted in fig. 10.

A far field calculation example is shown for the electromagnetic field generated by a $\lambda / 2$-dipole. The left metallic stub is coated with a material with the parameters $\epsilon_{\mathrm{r}}=2, \mu_{\mathrm{r}}=2$. The dipole is excited by a current connecting both metallic stubs. Figure 11 shows an arrowplot of the electric field. The far field characteristic can be seen in fig. 12 .

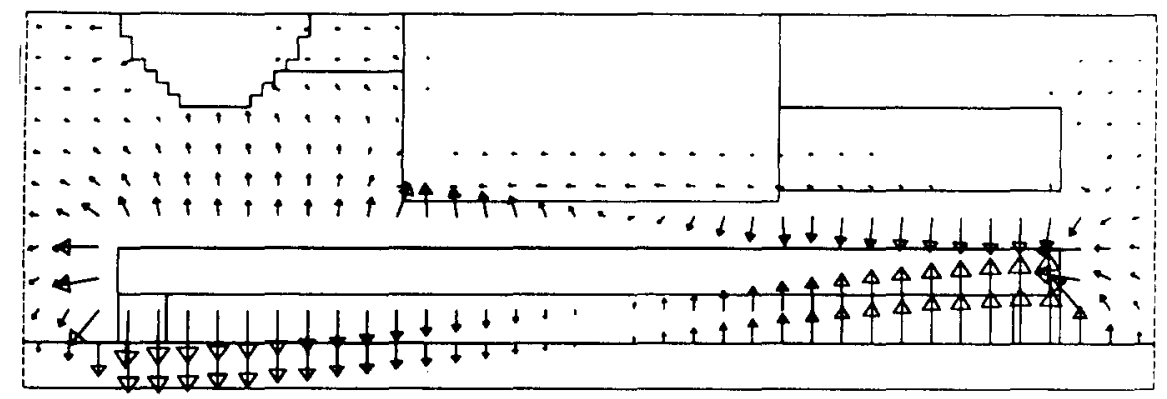

Fig. 14. Arrow plot of electric field in a cross-section of the nuclear resonance spectrometer. (Due to symetry the half structure was calculated.)

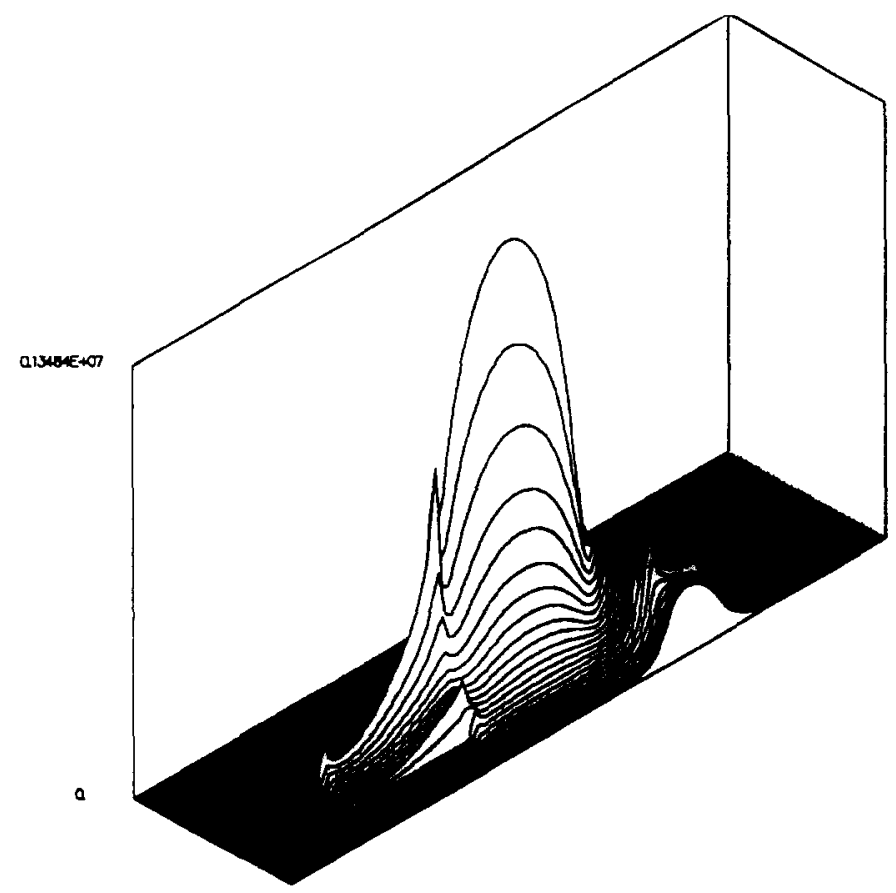

Fig. 15. Absorbed power density in the same cross-section as in the above figure. 
The last application examined the influence of a human test body in a three-dimensional nuclear resonance spectrometer, as seen in fig. 13. The rf stimulation structures are the two strip lines, mounted on dielectric supports at each end, on either side of the test person. Figures 14 and 15 show the calculated field ( $\mathrm{T} 3$ [12]) for a $90 \mathrm{MHz}$ driving current in a cut plane through the body and the antennas, first the electric field as an arrow plot, then the absorbed power density as a contour plot. The field of a $\lambda / 2$-antenna resonance can clearly be recognised. The weak field density within the body is a consequence of absorption and reflection caused by the conductivity and high permeability of biological tissue. Most of the power is absorbed at the part of the body closest to the antennas. The area of the neck shows a local maximum, which indicates body resonance.

\section{References}

[1] K.S. Yee, Numerical solution of initial boundary value problems involving Maxwell's equations in isotropic media, IEEE Trans. Antennas Propag. 14 (1966) 302.

[2] T. Weiland, A discretization method for the solution of Maxwell's equations for 6 component fields, AEU, Arch. für Elektron. und Übertragungstech. Electron. and Commun. 31 (1977) 116.

[3] T. Weiland, On the numerical solution of Maxwell's equations and applications in the field of accelerator physics, Particle Accel. 15 (1984) 245, and references therein.

[4] T. Weiland, On the unique solution of Maxwellian eigenvalue problems in three dimensions, Particle Accel. 17 (1985) 227.

[5] T. Weiland, Transient electromagnetic fields excited by bunches of charged particles in cavities of arbitrary shape, in: Proc. X1-th Int. Conf. on High Energy Accelerators, Geneva, 1980 (Birkhauser Verlag, Basel, 1980) p. 570.

[6] M. Bartsch et al., MAFIA release 3.X, in: Proc. 1990 Linear Accelerator Conference, Albuquerque, LA 12004-C, Los Alamos, p.372.

[7] K. Steinike, Calculation of the propagation characteristics of a circular waveguide excentrically loaded with a dielectric rod, Frequenz 44 (1990) 1.

[8] F. Krawczyk und Th. Weiland, A new static solver with open boundary conditions in the 3D-CAD system MAFIA, IEEE Trans. Magn. 22 (1988) 55.

[9] F. Krawczyk und Th. Weiland, 3D magneto- and electrostatic calculations using MAFIA-S3, in: Proc. 1st EPAC Conf., Rome, 1988 (World Scientific, Singapore, 1988) Vol 1, pp. 520-522.

[10] D. Schmitt and T. Weiland, IEEE Trans. Magn. 28 (1992) 1793.

[11] P. Hahne and T. Weiland, IEEE Trans. Magn. 28 (1992) 1801.

[12] M. Dehler, M. Dohlus and T. Weiland, IEEE trans. Magn. 28 (1992) 1797. 